\title{
Molecular Biology: A Milestone to Diagnosis of Vesiculobullous Lesion
}

\author{
Dr. Soumendu Bikash Maiti ${ }^{1}$, Dr. Nilotpol Kashyap ${ }^{2}$, Dr. Brij Kumar ${ }^{3}$, \\ Dr. Neelam Dewani ${ }^{4}$ \\ ${ }^{I}$ Senior Lecturer, Department of Oral Medicine and Radiology, \\ Pacific Dental College and Research Center, Udaipur \\ ${ }^{2}$ Professor and Head of the Department, Pedodontics and Preventive Dentistry, \\ Rungta College of Dental Sciences and Research, Bhilai \\ ${ }^{3}$ Post Graduate Student, Rungta College of Dental Sciences and Research, Bhilai \\ ${ }^{4}$ Post Graduate Student, Rungta College of Dental Sciences and Research, Bhilai
}

\begin{abstract}
Vesiculobullous lesion are group of disorders clinically manifests as formation of blisters which can either be vesicles and bulla. They pose a diagnostic challenge due to their similar or polymorphic clinical signs and symptoms and histological limitations and unpredictable progress of the lesions. Molecular biology which deals with the study of biology at molecular level provides a link between clinical and histological presentations. Pemphigus group of diseases are characterized by auto antibodies directed against desmosomal proteins. Pemphigoid group of lesions is characterized by circulating IgG autoantibodies against either BP 230 (BPAg1) and BP 180 (BPAg2) basement membrane zone proteins. Dermatitis herpetiformis is characterized by granular IgA deposits in dermal papiila whereas LINEAR IgA bullous dermatosis is characterized by IgA antibodies against $97 \mathrm{kDa}$ protein (LABD 97) AND $120 \mathrm{kDa}$ proteins. In epidermolysis bullosa aquisita autoantibodies are directed against type VII collagen fibres which is different from dystrophic epidermolysis bullosa characterized by mutation in type VII collagen.
\end{abstract}

Keywords: Vesiculobullous lesions, pemphigus, pemphigoid, dermatitis hepetiformis, Linear IgA bullous dermatosis, epidermolysis bullosa aquisita

\section{Introduction}

Mucocutaneous vesiculobullous lesions are affected by number of autoimmune and pathologic conditions. The proper treatment of these lesions relies on accurate diagnosis and understanding of the lesions at clinical, histopathological and molecular level. These lesions present themselves with diverse clinical signs and symptoms which poses a difficulty in diagnosing the lesion. ${ }^{1}$

Histological presentations has definite limitations due to a great polymorphism of these disorders. For eg: the acantholysis is recognized as a basic diagnostic criterion of pemphigus. along with "Tzanck test". But acantholysis may occur in some hereditable diseases (Hailey-Hailey disease, Darier's disease) and single acantholytic cells could be found in several diseases showing polymorphism nature of such lesions and histological limitations. ${ }^{2}$

Autoimmune disorders like pemphigus, pemphigoid etc are group of diseases characterized by immune mediated attack against body's self proteins or self antigens and these lesions usually are undetected before appearance of any clinical signs and symptoms and finally leading to destruction of epithelial structure. Molecular biology has put new insights into the understanding of these lesions at molecular level supplementing the histopathological findings and thereby devising the treatment plan based on this phenomenon. ${ }^{3}$

Moreover with the development of immunofluorescent techniques which includes both direct and indirect immunofluorescene molecular biology has resulted in the recognition of several new blistering diseases and classification based on clinical, histologic, and immunologic features. For understanding of the molecular biology understanding of the epithelial biology is quintessential. ${ }^{2}$

\section{Epithelial Biology (Desmosomes, Epithelial Basement Membrane Zone )}

Desmosomes and its proteins

They are anchoring junctions to which intermediate proteins like keratin filaments bind. The transmembrane proteins of desmosomes are desmogleins (Dsg) and desmocollins (Dsc) of cadherin family, desmoplakin (DP) and plakoglobin $(\mathrm{Pg})$, are the intracellular attachment protein in the desmosomes which mediate the interaction of keratin filaments with the cytoplasmic regions of desmocollins and desmogleins. (FIG $1 \& 2)^{4}$ 


\section{Structure Of Hemidesmosome And Basement Membarne Epithelial- Basement Membrane Zone}

The epithelial basement membrane zone (BMZ) is the zone consisting of epithelial basement membrane and its adjacent area along with hemidesmosomes which mediates keratinocyte-epithelial basement membrane contact. The BMZ can be divided into four zones from the epithelium towards the connective tissue consisting of various basement membrane zone proteins.( FIGURE $3 \& 4$, TABLE 1)

1. The keratinocytes plasma membrane with the hemidesmosomes which is further divided into inner and outer plaque

2. The outer lamina lucida zone

3. The inner lamina densa,.

b The sublamina densa ${ }^{5}$

\section{Pemphigus}

\section{Molecular Biology}

Pemphigus constitutes a group of chronic mucocutaneous blistering diseases where autoantibodies are directed against the desmosomal cadherin proteins desmoglein (Dsg)1 and/or Dsg characterized clinically by flaccid blisters and erosions of the skin and/or mucous membranes and microscopically by acantholysis microscopically by suprabasilar acantholysis. ${ }^{6}$ (FIG 5) In 1964 circulating antibodies was observed by BEUTNER and JORDAN when directed against the cell surface of keratinocytes in the sera of patients with Pemphigus vulgaris (PV) which further showed that these auto antibodies in pemphigus are pathogenic resulting in blister formation in skin organ culture systems and in neonatal mice. In 1982 Stanley et al. characterization of the PV antigen was conducted at the molecular level by immunoprecipitation techniques using cultured keratinocytes extracts as a substrate leading to identification of the PV sera as glycosylated 130 $\mathrm{kDa}$ glycoprotein. Two years later using immunoblot analyses of normal human epidermal extracts the pemphigus foliaceus (PF) antigen was characterized which further characterized that about one-third of the PF sera identified a polypeptide of about $160 \mathrm{kDa}$ protein.

This established the fact that patients with pemphigus have antibodies to desmosomal proteins. In pemphigus vulgaris patients, the antibodies are directed against the $130-\mathrm{kDa}$ desmosomal protein, and in pemphigus foliaceus patients against a $160-\mathrm{kDa}$ protein which was found to be the $\mathrm{N}$-terminal fragment of desmoglein-1.

Sequence analysis with the help of affinity- purified pemphigus vulgaris IgG, cDNA encoding pemphigus vulgaris antigen cloning from human keratinocyte expression libraries has revealed that the pemphigus vulgaris antigen is related, but not similar, to other desmogleins and is therefore termed desmoglein3 which has five extracellular domains, a transmembrane segment, and a cytoplasmic tail that interacts with the cytoskeleton. ${ }^{4}$ Pathogenic autoantibodies (IgG1 and IgG4) when directed against desmoglein 1 and desmoglein 3 it results in mucocutaneous involvement and when directed against against desmoglein 3 resulting in exclusive mucosal involvement. ${ }^{7}$

Several studies have also suggested a role for target antigen proteins other than desmogleins in pemphigus. Bedane et al. when compared the localization of immune deposits in patients with PV and PF by both direct and indirect immunoelectron microscopy found that in PV immune deposits both on the extracellular part of the desmosomes and along large portions of the keratinocytes without desmosomal structures in most of the studied samples, while in PF the immune deposits were located on the extracellular part of desmosomes only. Further study by Nguyen et al. demonstrated that injected Dsg3-lacking mice with PV, IgG that did not cross react with Dsg1 but uprabasal acantholysis and staining in a fishnet-like pattern was observed . leading to the hypothesis that mucocutaneous lesions in pemphigus could be caused by non desmoglein antibodies. ${ }^{8}$

Various theories of pathomechanism has been studied by which IgG induces acantholysis in pemphigus which are steric hindrance, deranged cell signaling, impairment of desmosome assembly and increased desmosome disassembly, Intracellular mechanism of acantholysis, Genetic factor, Epitope spreading and various Immune cells factors. ${ }^{7,8,9}$

\section{Bullous pemphigoid}

The disease bullous pemphigoid clinically manifests as tense blisters with and/or without erythema all over the body surface, histologically as subepithelial blister formation along with neutrophils with the split along the lamina lucida. ${ }^{10}$ (FIG 6)

The molecular pathogenesis of bullous pemphigoid (BP) is characterized by tissue bound and circulating IgG autoantibodies against two components of the hemidesmosomes i.e BP $230 \mathrm{kDa}(\mathrm{BPAg} 1)$ and $\mathrm{BP} 180 \mathrm{kDa}(\mathrm{BPAg} 2)$ and the noncollagenous 16A domain (NC16A), located at the membrane-proximal region of BP180, is thought to contain relevant epitopes of BP and is recognized by autoantibodies in $80-90 \%$ of BP patients. 
It also showed that autoantibodies preferentially recognize the phosphorylated BP180 ectodomain with recent studies have also demonstrated the presence of memory B cells which are specific for the NC16A domain. These BP180 NC16A-specific autoantibodies are not only of the IgG isotype (mainly IgG1 and IgG4 subclasses), but also IgE class have also been identified.

The circulating antibodies directed against the 230-kDa protein found in patients with bullous pemphigoid characterized by epiphenomenon, whereas the circulating antibodies against the 180-kDa protein are the pathogenic antibodies. The autoantibodies are first directed against

the $180-\mathrm{kDa}$ protein which damages the epidermal cells, allowing to access the 230- kDa protein and antibodies directed against it is thus formed. ${ }^{11}$

A strong evidence suggests the binding of pemphigoid antibodies to the basement membrane zone may be followed by complement activation responsible for inflammatory injury and blister formation. The response is dependent on the presence of leucocytes, complement, and bullous pemphigoid antibodies. The release of proteinases such as collagenases and elastases from neutrophils and eosinophils along with binding of BP 180 specific antibodies to their hemidesmosomal target antigen leads to blister formation. Various inflammatory mediators are released such as interleukins mostly IL-6 AND IL-8, tumour necrosis factor- $\alpha$ (TNF- $\alpha$ ), TNF- $\beta$, IFN- $\gamma$ and more eotaxin leading to the detachment of the basal cells from basement membrane zone and causing the dermal-epidermal splitting with complement mediated cell lysis. ${ }^{4,7}$

\section{Cicatrical pemphigoid/ mucous membrane pemphigoid}

In mucous membrane pemphigoid (MMP) patients BP180 is the target antigen in about $70 \%$ of the patients and the C-terminal epitopes of BP180 are preferentially targeted. MMP patients showed $\operatorname{IgG}$ and/or IgA autoantibodies of other specificity that recognize BP230, the 97/120kDa LAD antigen, laminin 332 (laminin 5), laminin 331 (laminin 6), type VII collagenor the $\alpha 6$ and $\beta 4$ integrin subunits. It has been shown that sera from patients with generalized MMP and ocular MMP recognize the $\beta 4$ integrin subunit, while sera from patients with oral MMP recognize the $\alpha 6$ integrin protein subunit.

Though MMP patients develop antibodies to the NC16A domain of BP180, Western blot technique revealed the BP180 reactivity react with LAD-1 or the C-terminal portion of BP180. The testing for IgA antiAssaying for anti-laminin 332 or laminin 5 antibodies is of utmost importance due development of malignancy in $25 \%$ of the patients. In these patients, a tumor search is indicated.

Direct IF microscopy reveals the linear deposition of IgG and $\mathrm{C} 3$ deposits in basement membrane zone . Indirect IF microscopy using $1 \mathrm{M} \mathrm{NaCl}$ split human skin is used to differentiate between antigens on the epithelial side of the split (BP180, BP230, and a6b4 integrin) and those of the dermal side (laminin 332).

The pathogenesis of the cicatricial pemphigoid lesions is by binding of auto-antibodies to BP 180 or laminin 5 and thereby activating the complement cascade and subsequently leading to the splitting of epithelium from underlying connective tissue. ${ }^{11}$

\section{Paraneoplastic pemphigus}

Paraneoplastic pemphigus (PNP) a severe variant of pemphigus characterized clinically by mucosal erosions, polymorphous skin eruptions, the presence of underlying neoplasia mostly non hodgkin's lymphoma, chronic lymphocytic leukemia and possess histological features of acantholysis and keratinocyte necrosis

A intercellular deposition of $\operatorname{IgG}$ as well basement membrane zone deposits of $\mathrm{IgG}$ or complement has been revealed by immunohistochemical investigations and the presence circulating $\operatorname{IgG}$ antibodies to cell surfaces of both stratified and non-stratified epithelia.

The complex of four epithelial proteins (250-kDa, 230-kDa, 210-kDa, and 190- kDa) has been demonstrated by immunoprecipitation out of which $250-\mathrm{kDa}$ and the $210-\mathrm{kDa}$ proteins

are desmoplakins I and II, and the $230-\mathrm{kDa}$ protein is the $230-\mathrm{kDa}$ bullous pemphigoid antigen, though the identity of the 190-kDa protein has not yet been established.

Studies using immunoelectron microscopy have shown presence of extracellular desmosomal antigens in PNP, including IgG4 autoantibodies to the $130-\mathrm{kDa}$ protein, which also reacted with serum from pemphigus vulgaris patients. ${ }^{4}$

\section{Dermatitis hepetiformis}

Dermatitis herpetiformis (DH), a blistering skin disease characterized by polymorphic rash with small blisters and intence itch on elbows, knees and buttocks. Upper back, abdomen, groins, axillae, and face are mostly affected but oral lesions are rare. Lesions can occur in any age and are often eroded and crusted because of intense itch and scratching along with complete remissions are unfrequent in a normal, gluten-containing diet. Granular IgA deposits in dermal papillae are the characteristic feature for DH but mostly seen in greater amounts with complement (C3) near the active lesions. Immunoelectron microscopical studies were the first to localize IgA deposits in close association with microfibrillar bundles of elastic fibers in the papillary dermis and 
in the dermoepidermal junction below the basal lamina.Blister formation starts with neutrophil micro-abscesses at the summits of dermal papillae which are quickly transformed by edema into micro-vesicles. They coalescence to form a unilocular subepidermal bulla and the split occurs in lamina lucida and the formation of blister formation takes about 24 hours. ${ }^{12}$

\section{Linear iga bullous dermatosis}

Linear IgA bullous dermatosis a mucocutaneous lesion mostly affecting the skin is which clinically resembles bullous pemphigoid or dermatitis herpetiformis. The histopathological features are similar to that seen in bullous pemphigoid with subepithelial bulla formation and neutrophil infiltration.In 1990 ZONE et al reported that immunoglobulin A ( $\operatorname{IgA})$ autoantibodies from patients with linear $\operatorname{IgA}$ disease react with a $97 \mathrm{kDa}$ protein (LABD97) of the DEJ. Marinkovich et al. in 1996 then identified a $120 \mathrm{kDa}$ protein (LAD1), synthesized by keratinocytes as the target of these IgA autoantibodies.In contrast to bullous pemphigoid, only $20 \%$ of sera of patients with LAD react with the NC16A domain. IgA antibodies to BP230, type VII collagen and laminin 332 have been reported. Further it was demonstrated that LABD97 and LAD-1 represent portions of the BP180 ectodomain resulting from cleavage within the membrane-proximal NC16A domain. Direct IF microscopy of perilesional skin shows linear deposition of $\operatorname{IgA}$ at the epidermal BMZ, often with linear IgG deposits. Though the circulating $\operatorname{IgA}$ autoantibodies mostly bind the epidermal side of $1 \mathrm{M} \mathrm{NaCl}$ split human skin on indirect IF microscopy (lamina lucida type), but combined epidermal and dermal staining as well as dermal labeling alone (sublamina densa type) has been demonstrated. ${ }^{4,11}$

\section{Epidermolysis bullosa acquisita ( eba )}

The term "epidermolysis bullosa acquisita" (EBA) is a chronic, autoimmune blistering disease characterized by a subepidermal blister formation occasionally associated with scarring and possess clinical features similar to those of dystrophic forms of hereditary epidermolysis bullosa, but without any clear cut inheritance pattern. They are of 2 types, the classic form, which results in a lesion of the basement membrane with little inflammation, and the the inflammatory form, which includes a significant infiltration of neutrophils. With the use of a panel of fusion proteins which corresponds to different segments of human type VII collagen for epitope mapping, it has been stated that several epitopes on the $\mathrm{N}$-terminal non-collagenous domain of type VII collagen are recognized by circulating autoantibodies in patients with epidermolysis bullosa acquisita. Direct immunoelectron microscopy have shown localization of immunodeposits to the lamina densa and sublamina densa zones which is complemented by Indirect immunoelectron microscopy studies which shows antibody binding to the lamina densa and the sub-lamina densa. The pathogenesis of blister formation is mediated by binding of the antibodies to the anchoring fibrils which activates the complement system. ${ }^{4}$

\section{Discussion And Conclusion}

The diagnosis of the blistering lesions diseases based on clinical presentation and histological features possess limitations due to a similar overlapping signs and symptoms and similar histological characteristics. Therefore with the advancement of knowledge of complex proteins and glycoproteins of basement membrane zones and detection of auto antibodies by different immunofloresence techniques had enlighten us with accurate recognition of these lesions and also also about pathogenesis of these lesions. Most of the autoimmune blistering diseases go undetected before the appearance of clinical and symptoms. With the help of molecular biology the autoantigens are characterized with their subcellular localization in basement membrane zone and to examining the effects of environmental agents on autoantigens expression, and to identify the antigenic epitopes as recognised by autoantibodies.

Moreover diagnostic criteria for subepidermal disorders were not accurate and different non acantholytic disorders were diagnosed as Duhring disease. Therefore dermatitis herpetiformis (DH) was regarded as a polymorphic disorder, sometimes with large blisters and divergent histopathologic findings. ${ }^{3,4}$

But with advent of molecular biology blistering lesions can be differentiated from each other and can be classified on basis of clinical, histological and immunologic features.

Epidermolysis bullosa aquisita (EBA) can be differentiated from DYSTROPHIC EPIDERMOLYSIS BULLOSA on basis of molecular biology such that former represents circulating autoantibodies against collagen VII or anchoring fibrils while the latter represents mutations of collagen VII.

Treatment modalities on the basis of generalized immunosuppression on the basis of past knowledge has not met with the success expected. Therefore, new strategies are based on immunomodulators rather than immunosuppression. Recent trends of clinical and molecular approaches involve vaccines, administration of antibodies and cytokines, gene therapy and use of antisense technologies. ${ }^{3}$

It is therefore has been quintessential to understand and study the molecular biology of the vesiculobullous lesion as the diagnosis of these lesions based on clinical presentation and histological patterns are not proper due to limitations and polymorphic character of these lesions. 
A formulation of a definitive diagnosis based on molecular biology pathogenesis will lead to produce a proper treatment plan treatment which could alleviate the signs and symptoms of the patient and give him a better life ahead.

Table 2: Showing Locations Of Various Basement Membrane Zone Proteins And Its Locations And Various Conditions Associated With It

\begin{tabular}{|l|l|l|}
\hline Proteins Of Basement Membrane & Also Known As & Bmz Location $^{\dagger}$ \\
\hline A 6 B 4 & & Transmembrane \\
\hline Bpag1 & Bp 230 & Intracellular \\
\hline Bpag 2 & Bp 180 & Transmembrane \\
\hline Laminin 5 & Epilgrin & \\
\hline Laminin 6 & & Bmz \\
\hline Keratin 5 & & Basal Layer Of Stratified Epithelia \\
\hline Keratin 14 & & Basal Layer Of Stratified Epithelia \\
\hline Collagen Type Vii & & Bmz \\
\hline Collagen Type Iv & & Bmz \\
\hline Ladinin & Lad 1 & Bmz \\
\hline
\end{tabular}

\section{References}

[1]. Nikitakis .G.N. Oral soft tissue lesions: A guide to differential diagnosis Part II: Surface alterations. Braz J Oral Sci. 2005 AprMay; 4 (13): 707-715.

[2]. Thivolet J, Jablonska S. Bullous Disorders: From Histology to Molecular Biology. Clinics in dermatology. 2001 Sept- Oct; 19 (5); 533-543.

[3]. Zahur M and Asif RA . Clinical, Cellular \& Molecular Biology of Autoimmune Disorders. J. Clin Cell Immunol. 2013

[4]. E. Dabeistee. Molecular biological aspects of acquired bullous diseases. Crit Rev Oral Biol Med. 9(2); 162-178.

[5]. Scully C, Bagan JV, Black M , Carrozzo M, Eisen D , Escudier M , Farthing P, Kuffer R , Muzio L , Mignogna M , Porter SR. Epithelial biology. Oral Diseases. 2005. 11; 58-7.

[6]. Tian J. Molecular Imaging: Fundamentals and applications. Beijing: Springer; 2013

[7]. Sangeetha S, Victor JD. The Molecular aspects of oral mucocutaneous diseases: a review. 2011 November; $3(10)$ : 141-148.

[8]. Wier Van de. Acantholysis in pemphigus. Netherlands; 2014.

[9]. Lanza A, Felice F, Fernando G. How acantholysis occur in pemphigus: a critical review. J Cutan Pathol. 2006 ; 13: 401-412.

[10]. Yasuo k. A new Perspective of Autoimmune Bullous Diseases: Molecular Cell Biology of Blistering Mechanisms and Logical Treatments. Dermatol Sinica. 2008; 26: 52-64.

[11]. Kasperkiewicz M , Zillikens D, Schmidt E. Pemphigoid diseases: Pathogenesis, diagnosis, and treatment . Autoimmunity February 2012; 45(1): 55-70.

[12]. Reunala LT . Dermatitis Herpetiformis. Clinics in dermatology. 2001; 19:728-736. 